\title{
Design of NPR-Type Cosine Modulated Filterbank Using Combinational Window Functions
}

\author{
Ram Kumar Soni ${ }^{1}$, Alok Jain ${ }^{2}$, Rajiv Saxena ${ }^{3}$ \\ ${ }^{1}$ Department of Electronics \& Communication Engineering, Samrat Ashok Technological Institute (Polytechnic), \\ Vidisha, India \\ ${ }^{2}$ Department of Electronics \& Instrumentation Engineering, Samrat Ashok Technological Institute, \\ Vidisha, India \\ ${ }^{3}$ Department of Electronics \& Communication Engineering, Jaypee University of Engineering \& Technology, \\ Raghogarh, Guna, India \\ E-mail: \{soniram04,alokjain6\}@rediffmail.com,rajiv.saxena@jiet.ac.in \\ Received September 8, 2010; revised October 10, 2010; accepted November 18, 2010
}

\begin{abstract}
This paper presents the design of near perfect reconstruction (NPR) cosine modulated filterbank (CMFB). The prototype filter is designed by the combinational window functions. These window functions provide high side-lobe-fall-off-rate (SLFOR) with better far-end attenuation which suppresses the undesired interferences occur in the filterbank. A linear optimization is used to minimize the error parameters. Design examples have been included to illustrate the effectiveness of the proposed technique over the earlier reported work.
\end{abstract}

Keywords: Combinational Window Functions, SLFOR, Far-End Attenuation, Stopband Energy

\section{Introduction}

Cosine modulated filterbank are used in a wide range of applications, from data compression of speech, audio and video signals to data transmission [1-6]. They are special subclass of the general M-channel filterbank, which has some very attractive features, first, it is a highly selective and discrimination system. Second, all of the analysis $H_{z}(z)$ and synthesis $F_{z}(\mathrm{z})$ filters of filterbank can be simultaneously generated by the cosine modulation of single linear phase FIR prototype filter as shown in Figure 1. Thirdly, the error occurs at the reconstructed output in NPR types system can easily optimized by using suitable optimization technique [2,7]. The CMFB has been studied by many prominent authors in PR and NPR conditions and finds that the NPR type is simple, more realizable and computationally efficient [3-9]. Also the performance of the system can be further improved with high stopband attenuation and narrow transition bandwidth of the prototype filter [8].

In speech and audio communication the stopband attenuation $\left(\Delta_{s}\right)$ of $40-60 \mathrm{~dB}$ provides the signals of adequate quality if the crosstalk suppression is efficient $[10,11]$. It mainly depends on the different parameters of prototype filter, i.e., stopband energy $\left(E_{s}\right)$, far-end attenuation $\left(\Delta_{f}\right)$ and SLFOR $[2,10]$. A window function with minimum stopband energy, better far-end attenuation and high SLFOR is the most suitable in such applications. Combinational window functions provide all these characteristics. These are designed by combining a data window and a lag window function in a linear manner $[2,10]$.

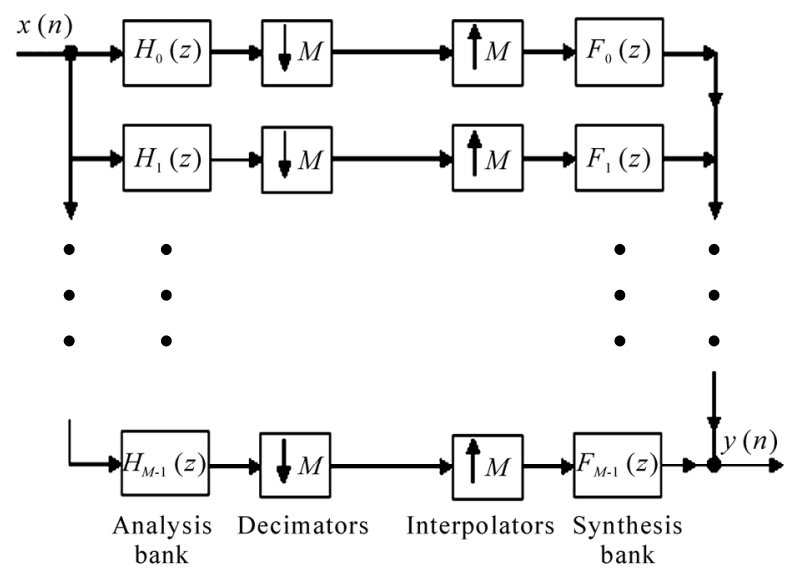

Figure 1. $M$-band cosine modulated filterbank. 
In this proposed work 8-, 16-, 32-band NPR CMFB is designed. The prototype FIR filter is formulated using Parzen-Cos ${ }^{6}$ (PC6) and Papoulis-Cos ${ }^{4}$ (PC4) combinational window functions $[10,12]$. The main contribution of this paper is summarized below:

1) The prototype filter is designed using high SLFOR combinational window functions.

2) The NPR CMFB is designed using cosine modulation.

3) The reconstructed output is not exact replica of the input. A linear optimization is applied in order to approximate the power complementary property.

4) Examples are included to evaluate the performance of the proposed technique in terms of error parameters, far-end attenuation and stopband energy.

\section{Design of Prototype Filter}

The impulse response coefficients of a causal $N$ th-order linear phase FIR filter $p[n]$ using window technique is given by [2]:

$$
p[n]=w[n] h[n]
$$

where, $h[n]$ is the impulse response of the ideal lowpass filter and is expressed as:

$$
h[n]=\frac{\sin \left(\omega_{\mathrm{c}}(n-0.5 N)\right)}{\pi(n-0.5 N)}
$$

where, $\left(\omega_{c}\right)$ is the cutoff frequency of the ideal lowpass filter. $w[n]$ is the used window function. PC6 and PC4 combinational window functions are used to design prototype filter. These window functions are characterized for high SLFOR and far-end attenuation [10]. The value of SLFOR for PC4 and PC6 window functions are $-24 \mathrm{~dB} /$ octave to $-30 \mathrm{~dB} /$ octave, $-24 \mathrm{~dB} /$ octave to $-42 \mathrm{~dB} /$ octave, respectively $[10,12]$. Whereas this figure for Kaiser window is only $-6 \mathrm{~dB} /$ octave [2]. The expressions for these window functions in time domain are given below $[10,12]$.

\subsection{Parzen- $\operatorname{Cos}^{6}(n \pi / N)$ (PC6) Combinational Window}

The expression for Parzen (lag window, $l(n)$ ) and $\operatorname{Cos}^{6}(n \pi / N)$ (data window, $\left.d(n)\right)$ combinational window with $\gamma_{6}$ as window shape parameter is given as $[10,12]$

$$
w_{P C 6}(n)=\left\{\begin{array}{cl}
\gamma_{6}\left[l_{6}(n)\right]+\left(1-\gamma_{6}\right)\left[d_{6}(n)\right], & |n| \leq \frac{N}{2} \\
0, & |n|>\frac{N}{2}
\end{array}\right.
$$

where $0 \leq \gamma_{6} \leq 3.7$ and

$$
\begin{gathered}
l_{6}(n)=\left\{\begin{array}{c}
1-24\left|\frac{n}{N}\right|^{2}\left(1-2\left|\frac{n}{N}\right|\right), \quad|n|<\frac{N}{4} \\
2\left(1-2\left|\frac{n}{N}\right|\right)^{3}, \quad \frac{N}{4} \leq|n| \leq \frac{N}{2}
\end{array}\right. \\
d_{6}(n)=\cos ^{6}\left(\frac{n \pi}{N}\right), \quad|n| \leq \frac{N}{2}
\end{gathered}
$$

FIR filter design relationships are given by the following equations [10]:

1) Relationship between window shape parameter $\left(\gamma_{6}\right)$ and desired stopband attenuation $\left(\Delta_{s}\right)$ :

$$
\gamma_{6}=a+\left(b \Delta_{s}\right)+\left(c \Delta_{s}^{2}\right)
$$

where,

$$
\begin{gathered}
a=8.15414, \quad b=-0.236709, \quad c=0.00218617 \\
\text { for } 30.32 \leq \Delta_{s} \leq 51.25 \\
a=21.3669, \quad b=-0.605789, \quad c=0.00434808 \\
\text { for } 51.25<\Delta_{s} \leq 68
\end{gathered}
$$

2) Relationship between normalized window width parameter $(D)$ and $\left(\Delta_{s}\right)$ :

$$
D=a+\left(b \Delta_{s}\right)+\left(c \Delta_{s}^{2}\right)
$$

where,

$$
\begin{gathered}
a=1.82892, \quad b=-0.0275481, \quad c=0.00157699 \\
\text { for } 30.32 \leq \Delta_{s} \leq 43.60 \\
a=1.67702, \quad b=-0.0450205, \quad c=0 \\
\text { for } 43.60<\Delta_{s} \leq 49.44 \\
a=85.4738, \quad b=-3.41969, \quad c=0.035784 \\
\text { for } 49.44<\Delta_{s} \leq 57.48 \\
a=-8.60006, \quad b=0.477004, \quad c=-0.00355655 \\
\text { for } 57.48<\Delta_{s} \leq 68.69
\end{gathered}
$$

\subsection{Papoulis-Cos ${ }^{4}(n \pi N)$ (PC4) Combinational Window}

The combinational window of Papoulis (lag window, $l(n)$ ) and $\operatorname{Cos}^{4}(n \pi / N)$ (data window, $\left.d(n)\right)$ with $\gamma_{4}$ as combinational factor is given by [12]:

$$
w_{P C 4}(n)=\left\{\begin{array}{cc}
\gamma_{4}\left[l_{4}(n)\right]+\left(1-\gamma_{4}\right)\left[d_{4}(n)\right], & |n| \leq \frac{N}{2} \\
0, & |n|>\frac{N}{2}
\end{array}\right.
$$




$$
\begin{gathered}
l_{4}(n)=\frac{1}{\pi}\left|\sin \left(\frac{2 \pi n}{N}\right)\right|+\left(1-2\left|\frac{n}{N}\right|\right) \cos \left(\frac{2 \pi n}{N}\right),|n| \leq \frac{N}{2} \\
d_{4}(n)=\cos ^{4}\left(\frac{n \pi}{N}\right),|n| \leq \frac{N}{2}
\end{gathered}
$$

FIR filter design relationships for PC4 window are given by the following equation [12].

1) Relationship between window shape parameter $\left(\gamma_{4}\right)$ and desired stopband attenuation $\left(\Delta_{s}\right)$ :

$$
\begin{gathered}
\gamma_{4}=a+\left(b \Delta_{s}\right)+\left(c \Delta_{s}^{2}\right)+\left(d \Delta_{s}^{3}\right)+\left(e \Delta_{s}^{4}\right) \\
\text { for } 26.19<\Delta_{s} \leq 61.08
\end{gathered}
$$

where, $a=-69.058755, b=8.409918, c=-0.321364, d$ $=0.005044 ; e=-0.000028$.

2) Relationship between normalized window width parameter $(D)$ and $\left(\Delta_{s}\right)$ :

$$
\begin{gathered}
D=a+\left(b \Delta_{s}\right)+\left(c \Delta_{s}^{2}\right)+\left(d \Delta_{s}^{3}\right)+\left(e \Delta_{s}^{4}\right), \\
\quad \text { for } 26.19<\Delta_{s} \leq 61.08
\end{gathered}
$$

where, $a=8.728537, b=-0.412899, c=-0.000713, d=$ $0.000355 ; e=-0.000004$.

The order $(N)$ of the filter designed by using above mentioned window functions can be estimated by the following formula:

$$
N=\left\lceil\frac{D}{\Delta \omega}\right\rceil+1
$$

where, $D$ is the window width parameter and $\Delta \omega$ is the normalized transition width $=\left(\omega_{s}-\omega_{p}\right) / 2 \pi$ with $\omega_{s}$ and $\omega_{p}$ are the stopband and passband frequencies, respectively. $\lceil x\rceil$ represents the smallest integer greater than or equal to $x$.

\section{Cosine Modulation}

Cosine modulation is one of the efficient techniques which provide minimum computational cost in the design of filterbank. All filters of synthesis and analysis sections are obtained by cosine modulation of a linear phase lowpass prototype filter $[2,13]$.

$$
\left.\begin{array}{l}
h_{k}(n)=2 p(n) \cos \left[(2 k+1) \frac{\pi}{2 M}\left(n-\frac{N}{2}\right)+(-1)^{k} \frac{\pi}{4}\right] \\
f_{k}(n)=2 p(n) \cos \left[(2 k+1) \frac{\pi}{2 M}\left(n-\frac{N}{2}\right)-(-1)^{k} \frac{\pi}{4}\right]
\end{array}\right\}
$$$$
\text { for } 0 \leq k \leq M-1 \text {, and } 0 \leq n \leq N
$$

where, $M$ is the number of bands and $h_{k}(n)$ and $f_{k}(n)$ are the impulse responses of the analysis and synthesis sections, respectively.

\section{Optimization Technique}

In NPR system, the PR condition is relaxed by allowing small amount of errors. There are three types of errors occur at the reconstructed output, as follows amplitude $\left(E_{p p}\right)$, phase and aliasing $\left(E_{a}\right)[2,10]$. The measures of these error parameters are given by the following equations:

$$
\begin{aligned}
& \left|P\left(e^{j \omega}\right)\right| \approx 0 \text { for }|\omega|>\pi / M \\
& T_{o}\left(e^{j \omega}\right) \approx 1, \\
& \text { where } T_{o}\left(e^{j \omega}\right)=\sum_{k=0}^{2 M-1}\left|P\left(e^{j(\omega-k \pi / M)}\right)\right|^{2}
\end{aligned}
$$

is the overall distortion transfer function. The accuracy of first approximation gives aliasing and the accuracy of the second one gives the reconstruction error. The phase error is eliminated by linear phase prototype filter. However, other two distortion parameters can be minimized by applying suitable optimization technique. Much of work have been done in this field $[10,11,14,15]$. Initially, Johnston [15] developed a nonlinear optimization technique, later on many prominent authors such as Creusere et al. [11] and Lin et al. [14] and Jain et al. [10] have simplified it and developed single variable linear optimization techniques with different objective functions. In the proposed work, a linear gradient optimization technique is used with objective function given in Equation (13). The cutoff frequency of prototype filter is varied to obtain the minimum value of the objective function.

$$
\begin{aligned}
& \phi=\left.\max || P\left(e^{j \omega}\right)\right|^{2}+\left.P\left(e^{j(\omega-\pi / M)}\right)\right|^{2}-1 \mid \\
& \text { for } 0 \leq \omega<\pi / M
\end{aligned}
$$

Initially, input parameters, i.e., sampling rate, number of band, passband and stopband frequencies, passband ripple and stopband attenuation of prototype filter are specified and determine the cutoff frequency, transition band and filter length. Initialize, different optimization pointers like step size, search direction, flag and initial (p_err) as well as expected minimum possible values (e_err) of objective function. Inside the optimization loop, design the prototype lowpass filter and determine the bandpass filters for analysis and synthesis sections using cosine modulation. In optimization routine cutoff frequency is gradually changed as per the search direction and calculates the corresponding value of the objective function. Algorithm halts when it attains the minimum value of the objective function. The flowchart of optimization is given in appendix section and simulated on MATLAB 7.0. 


\section{Design Examples}

The performance evaluation of the proposed technique has been made with the help of design examples. It is examined in terms of stopband attenuation, reconstruction error, aliasing error, computational complexity, group delay and stopband energy. The effectiveness of the proposed work is compared with the earlier reported work for the same input parameters.

Example 1: An eight-band CMFB has been designed using PC6 and PC4 window functions. Same specifica- tions are taken as given by Kha et al. [16], i.e., stopband attenuation $\left(\Delta_{\mathrm{s}}\right)=35.8 \mathrm{~dB}$ and stopband frequency $\left(\omega_{s}\right)=0.12 \pi$, respectively. The frequency response of prototype FIR filter, eight-band CMFB and the magnitude responses of reconstruction and aliasing errors for PC6 and PC4 window functions are shown in Figures 2 (a)-(d) and 3(a)-(d), respectively.

The obtained values of reconstruction error, aliasing error, far-end attenuation, stopband energy are given in Table 1.

Example 2: In this example, sixteen-band CMFB is

Table 1. Performance comparison with Kha et al. [16].

\begin{tabular}{|c|c|c|c|c|c|c|c|c|c|}
\hline Work & Window function & M & $\Delta_{s}(\mathrm{~dB})$ & $\omega_{s}$ & $N$ & $E_{p p}$ & $E_{a}$ & $E_{s}$ & $\Delta_{f}$ \\
\hline \multirow{3}{*}{ Kha et al. [16] } & \multirow{3}{*}{ Kaiser } & 8 & 35.8 & $0.12 \pi$ & 40 & $5.50 \times 10^{-3}$ & $2.47 \times 10^{-3}$ & $1.17 \times 10^{-2}$ & 50 \\
\hline & & 16 & 45 & $0.059 \pi$ & 102 & $5.95 \times 10^{-3}$ & $3.89 \times 10^{-4}$ & $1.46 \times 10^{-4}$ & 70 \\
\hline & & 32 & 102 & $0.031 \pi$ & 466 & $9.12 \times 10^{-4}$ & $2.38 \times 10^{-7}$ & $1.04 \times 10^{-6}$ & 120 \\
\hline \multirow{5}{*}{ Proposed } & $\mathrm{PC} 4$ & 8 & 35.8 & $0.12 \pi$ & 47 & $5.79 \times 10^{-3}$ & $7.42 \times 10^{-4}$ & $1.05 \times 10^{-2}$ & 110 \\
\hline & PC6 & 8 & 35.8 & $0.12 \pi$ & 49 & $2.60 \times 10^{-3}$ & $6.25 \times 10^{-3}$ & $8.60 \times 10^{-3}$ & 105 \\
\hline & PC4 & 16 & 45 & $0.059 \pi$ & 151 & $4.04 \times 10^{-4}$ & $3.20 \times 10^{-4}$ & $3.05 \times 10^{-5}$ & 137 \\
\hline & PC6 & 16 & 45 & $0.059 \pi$ & 123 & $7.25 \times 10^{-4}$ & $4.85 \times 10^{-5}$ & $1.41 \times 10^{-5}$ & 140 \\
\hline & PC6 & 32 & 61 & $0.031 \pi$ & 370 & $8.93 \times 10^{-4}$ & $6.77 \times 10^{-4}$ & $2.06 \times 10^{-7}$ & 160 \\
\hline
\end{tabular}

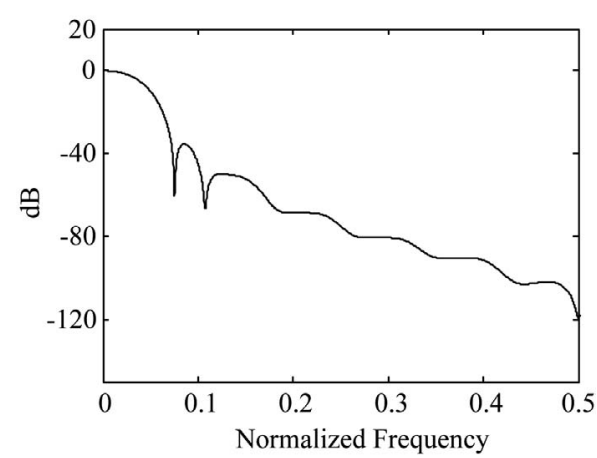

(a)

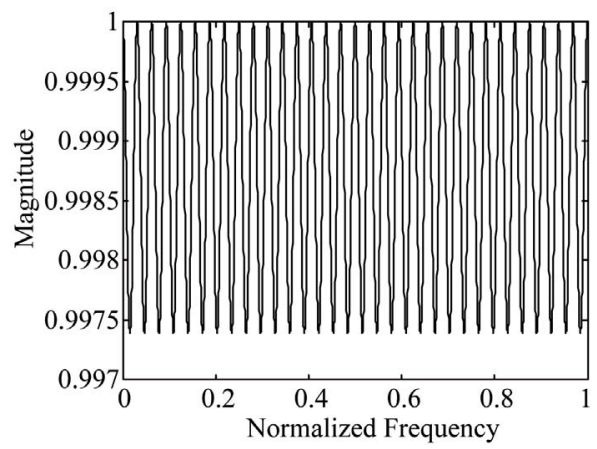

(c)

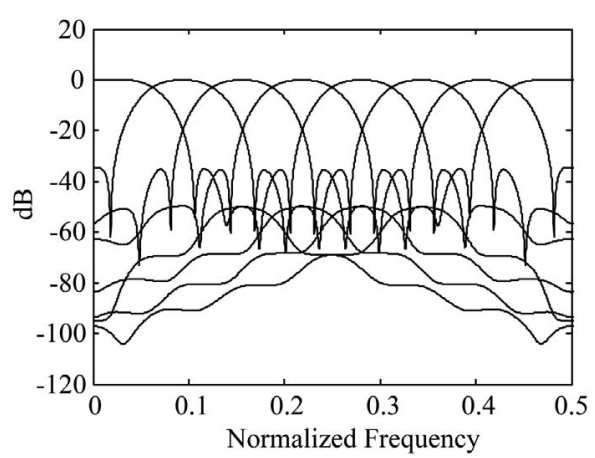

(b)

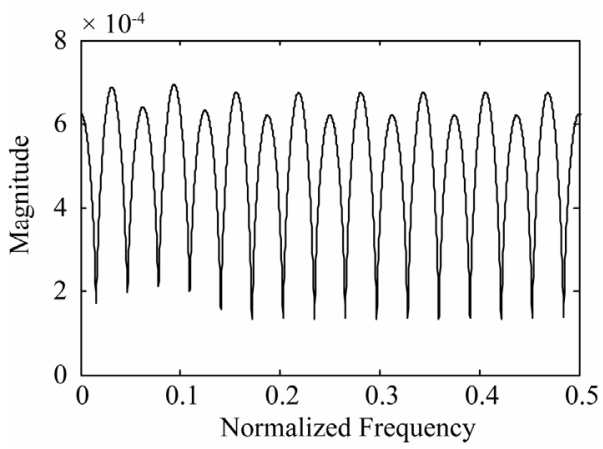

(d)

Figure 2. Magnitude responses of 8-band CMFB using PC-6 window at $\Delta_{s}=35.8 \mathrm{~dB}$. (a) Prototype lowpass filter; (b) Filterbank; (c) Plot of reconstruction error; (d) Plot of aliasing error. 


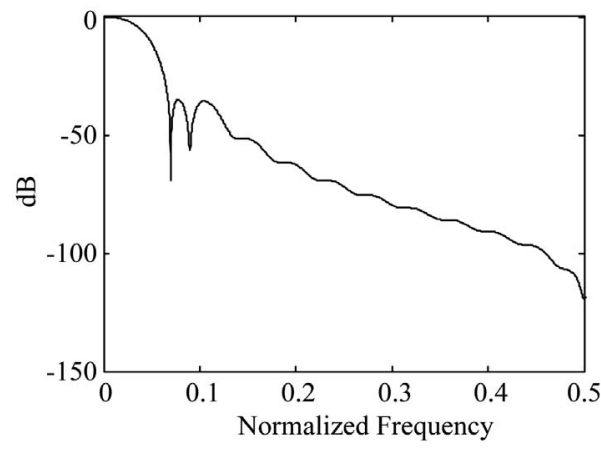

(a)

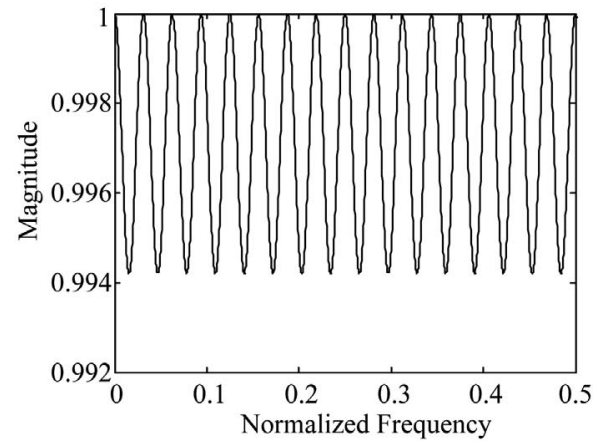

(c)

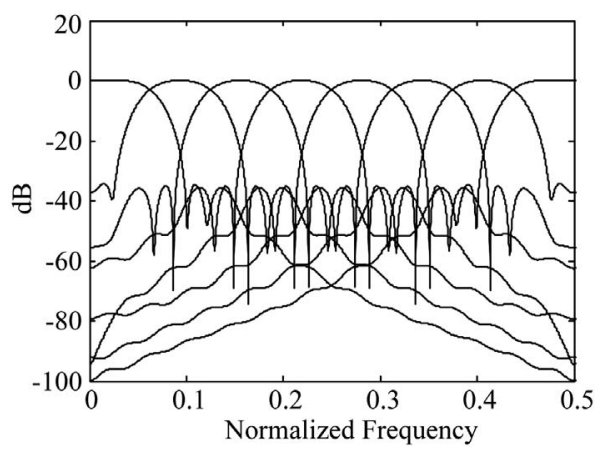

(b)

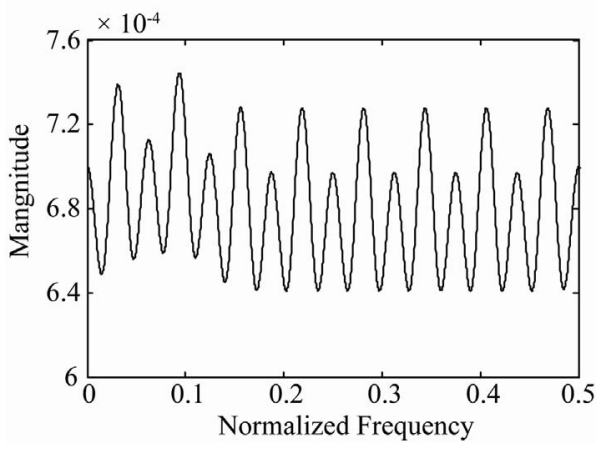

(d)

Figure 3. Magnitude responses of 8-band CMFB using PC-4 window at $\Delta_{s}=35.8 \mathrm{~dB}$. (a) Prototype lowpass filter; (b) Filterbank; (c) Plot of reconstruction error; (d) Plot of aliasing error.

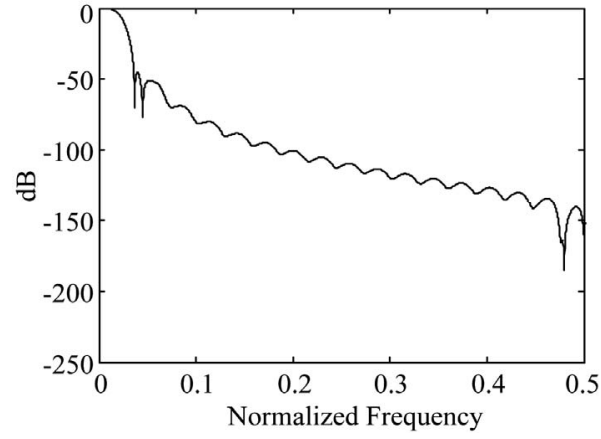

(a)

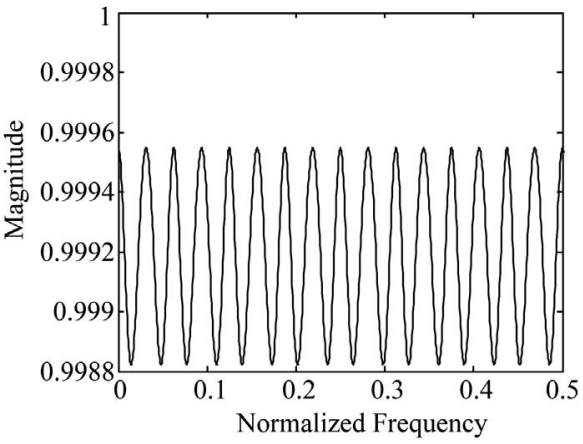

(c)

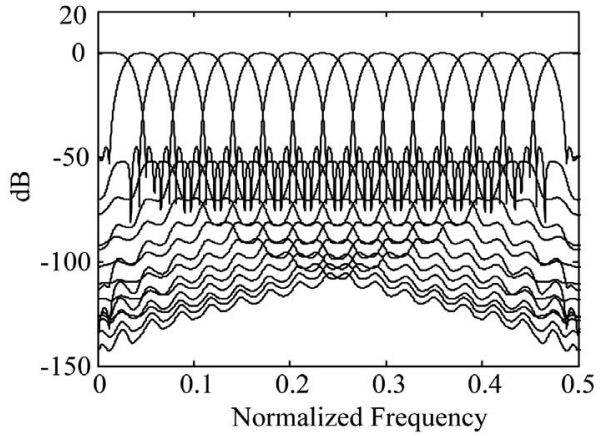

(b)

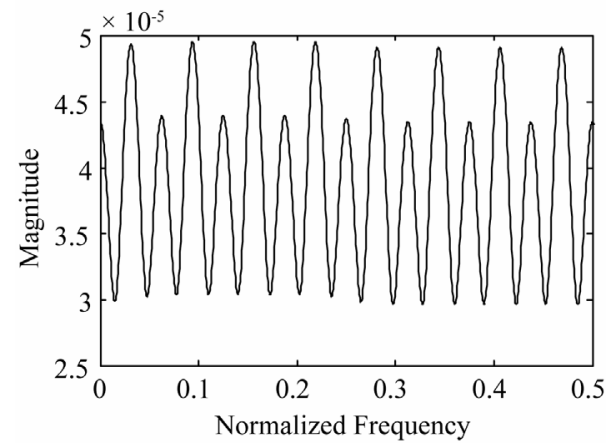

(d)

Figure 4. Magnitude responses of 16-band CMFB using PC6 window at $\Delta_{s}=45 \mathrm{~dB}$. (a) Prototype lowpass filter; (b) Filterbank; (c) Plot of reconstruction error; (d) Plot of aliasing error. 
designed using same specifications as given in [16]. The stopband attenuation and stopband frequency are taken as $\left(\Delta_{s}\right)=45 \mathrm{~dB},\left(\omega_{s}\right)=0.0590 \pi$, respectively. The magnitude responses of prototype FIR filter, 16-band CMFB, reconstruction error and aliasing error for both window functions are shown in Figure 4 and Figure 5, respectively. The optimized value of performance parameters are given in Table 1.

Example 3: A thirty two-band CMFB has been designed using PC6 window function with the same stopband frequency as taken by Kha et al. [16]. Since, the stopband attenuation for PC6 window functions is restricted up to $68.69 \mathrm{~dB}$, therefore, $61 \mathrm{~dB}$ stopband attenuation is taken for the design. The frequency responses are shown in Figures 6(a)-(d), respectively. The obtained values of different parameters are given in Table 1.

\section{Discussion}

The comparative performance of proposed work with the reported publication is given in Table 1. In case of 8-band CMFB the obtained values of reconstruction error for PC4 and PC6 window functions is $5.79 \times 10^{-3} ; 2.60 \times$

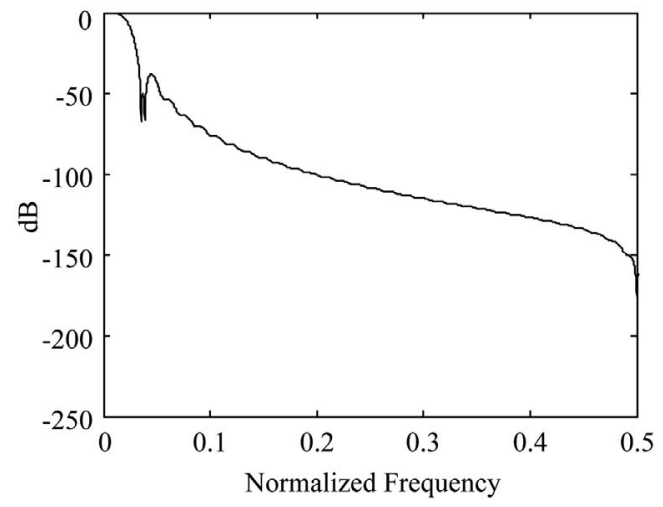

(a)

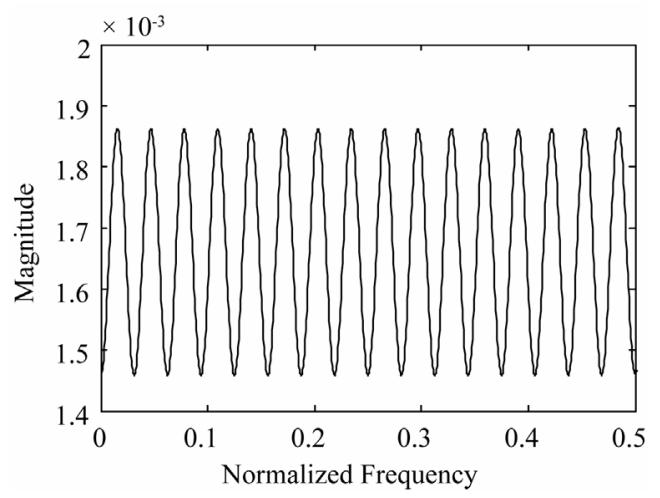

(c)
$10^{-4}$, respectively, which is smaller than the reported value of Kha et al. [16]. Similarly, the aliasing error in reported and proposed work is $2.47 \times 10^{-3}$ and $7.42 \times$ $10^{-4}, 6.25 \times 10^{-3}$, respectively. Apart from lower values of error parameters the proposed prototype filter using combinational window functions are providing reduction in stopband energy and better far-end attenuation in contrast to Kha et al.

Similarly, in case of 16-band CMFB the value of error parameters are much smaller than the Kha et al. [16] with smaller value of stopband energy and more far-end attenuation. In 32-band CMFB the proposed technique provides smaller value of reconstruction error than the existing journal with less stopband energy and better far-end attenuation.

\section{Conclusions}

An efficient design for M-band NPR CMFBs has been proposed using combinational window functions. Simulation studies shows that the developed algorithm provides smaller values of error parameters than the previous publication. High values of SLFOR and far-end attenuation

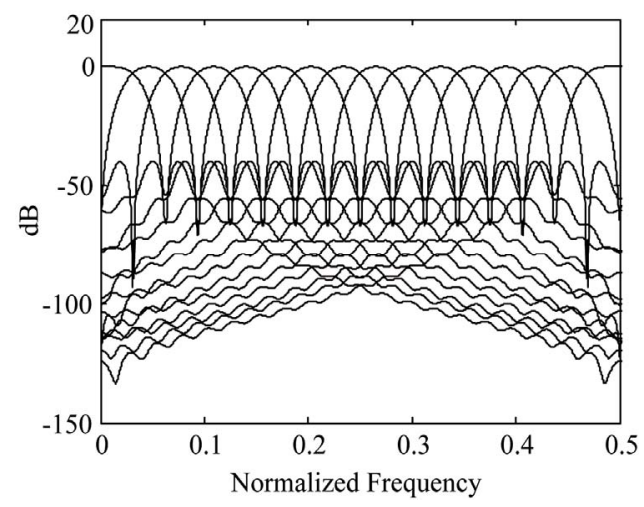

(b)

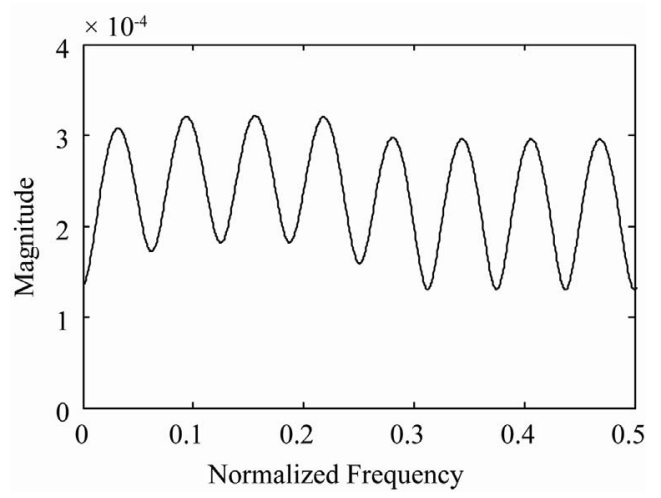

(d)

Figure 5. Magnitude responses of 16-band CMFB using PC4 window at $\Delta_{s}=45 \mathrm{~dB}$. (a) Prototype lowpass filter; (b) Filterbank; (c) Plot of reconstruction error; (d) Plot of aliasing error. 


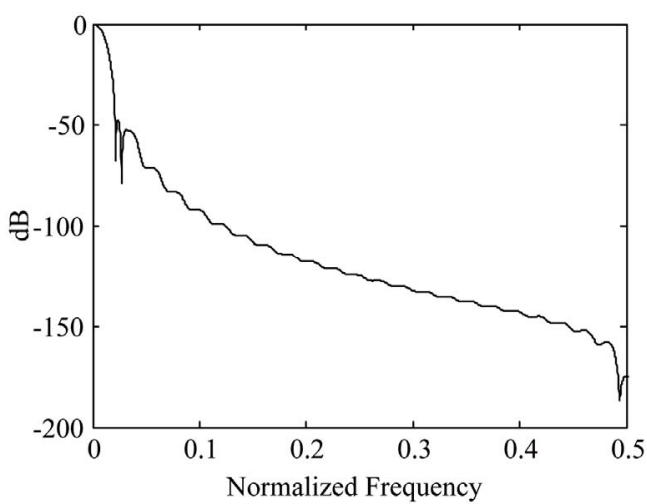

(a)

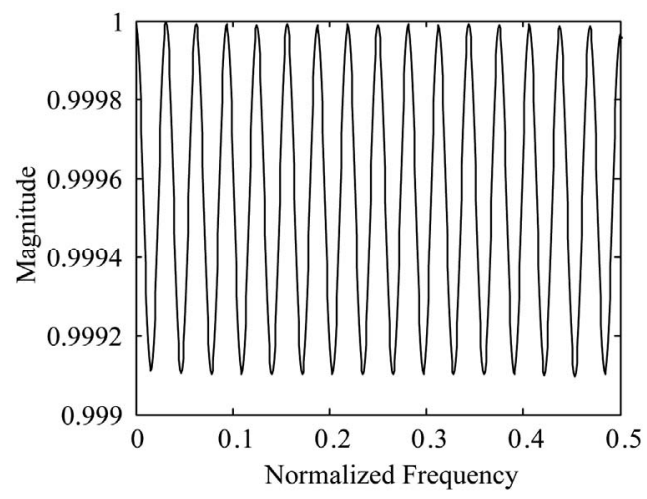

(c)

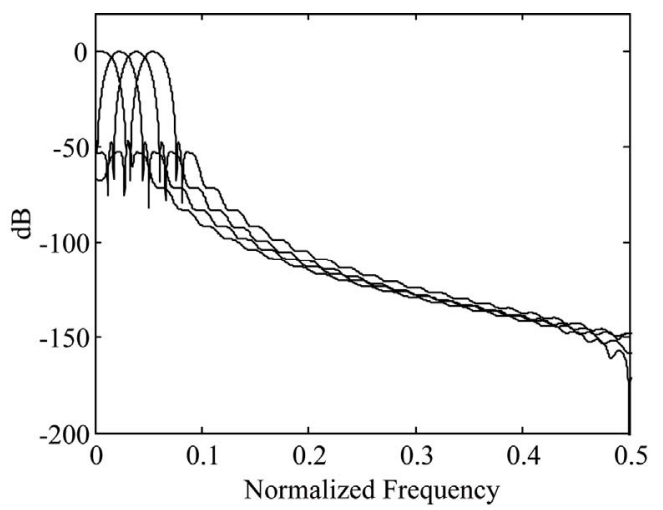

(b)

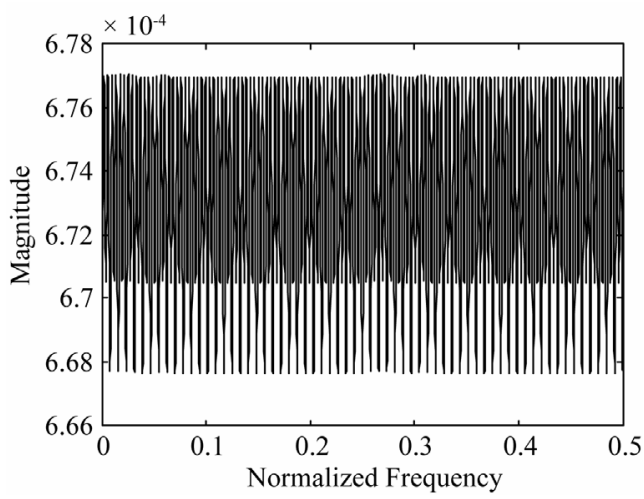

(d)

Figure 6. Magnitude responses of 32-band CMFB using PC-6 window at $\Delta_{s}=61 \mathrm{~dB}$. (a) Prototype lowpass filter; (b) Filterbank; (c) Plot of reconstruction error; (d) Plot of aliasing error.

provide significant reduction in stopband energy. These filterbank can be used in real time applications such as echo cancellation and cross-talk suppression.

\section{References}

[1] L. Lin and B. Farhang-Boroujeny, "Cosine-Modulated Multitone for very High Speed Digital Subscribe Lines," EURASIP Journal on Applied Signal Processing, Vol. 2006, 2006, p. (19329)79.

[2] P. P. Vaidyanathan, "Multirate Systems and Filter Banks," Prentice-Hall, Englewood Cliffs, 1993.

[3] W. S. Lu, T. Saramäki and R. Bregovic, "Design of Practically Perfect-Reconstruction Cosine-Modulated Filter Banks: A Second-Order Cone Programming Approach," IEEE Transactions on Circuits and System-I, Vol. 51, No. 3, May 2004, pp. 552-563.

[4] M. B. Furtado, P. S. R. Diniz and S. L. Netto, "Numerically Efficient Optimal Design of Cosine Modulated Filter Banks with Peak-Constrained Least-Squares Behavior," IEEE Transactions on Circuits and System-I, Vol. 52, No. 3, May 2005, pp. 597-608.

[5] M. B. Furtado, P. S. R. Diniz, S. L. Netto, and T. Saramäki, "On the Design of High-Complexity Cosine-Modulated
Transmultiplexers Based on the Frequency-Response Masking Approach," IEEE Transactions on Circuits and System-I, Vol. 52, No. 11, November 2005, pp. 24132426.

[6] M. Blanco-Velasco, F. Cruz-Roldán, E. Moreno-Martínez, J. I. Godino and K. E. Barner, "Embedded Filter BankBased Algorithm for ECG Compression," Signal Processing, Vol. 88, No. 6, June 2008, pp. 1402-1412.

[7] F. Cruz-Roldán and M. Monteagudo, "Efficient Implementation of Nearly-Perfect Reconstruction Cosine-Modulated Filterbanks," IEEE Transactions on Signal Processing, Vol. 52, No. 9, September 2004, pp. 2661-2664.

[8] T. Q. Nguyen, "Near-Perfect-Reconstruction Pseudo QMF Banks," IEEE Transactions on Signal Processing, Vol. 42, No. 1, January 1994, pp. 65-76.

[9] C. K. Goh and Y. C. Lim, "Design of Two Channel Perfect Reconstruction Linear Phase FIR Filterbanks," IEEE Transactions on Circuits and System-II: Analog and Digital Signal Processing, Vol. 45, August 1998.

[10] A. Jain, R. Saxena and S. C. Saxena, "An Improved and Simplified Design of Cosine Modulated Pseudo-QMF Filterbanks," Digital Signal Processing, Vol. 16, No. 3, May 2006, pp. 225-232.

[11] C. D. Creusere and S. K. Mitra, "A Simple Method for Designing High-Quality Prototype Filters for M-Band 
Pseudo QMF Banks," IEEE Transactions on Signal Processing, Vol. 43, No. 4, April 1995, pp. 1005-1007.

[12] S. N. Sharma, R. Saxena and S. C. Saxena, "Design of FIR Filters Using Variable Window Families: A Comparative Study," Journal of Indian Institute of Science, Vol. 84, October 2005, pp. 155-161.

[13] S. K. Mitra, "Digital Signal Processing-A Computer Based Approach," Tata McGraw-Hill Publishing Co. Ltd., New Delhi, 1998.

[14] Y. Lin and P. P. Vaidyanathan, "A Kaiser Window Approach for the Design of Prototype Filters of Cosine
Modulated Filterbanks," IEEE Signal Processing Letters, Vol. 5, No. 6, June 1998, pp. 132-134.

[15] J. D. Johnston, “A Filter Family Designed for Use in Quadrature Mirror Filter Banks," Proceedings of IEEE International Conference on Acoustics, Speech and Signal Processing, Denver, 9-11 April 1980, pp. 291-294.

[16] H. H. Kha, H. D. Tuan and T. Q. Nguyen, "Efficient Design of Cosine-Modulated Filterbanks via Convex Optimization," IEEE Transactions on Signal Processing, Vol. 57, No. 3, 2009, pp. 966-976. 


\section{Appendix}

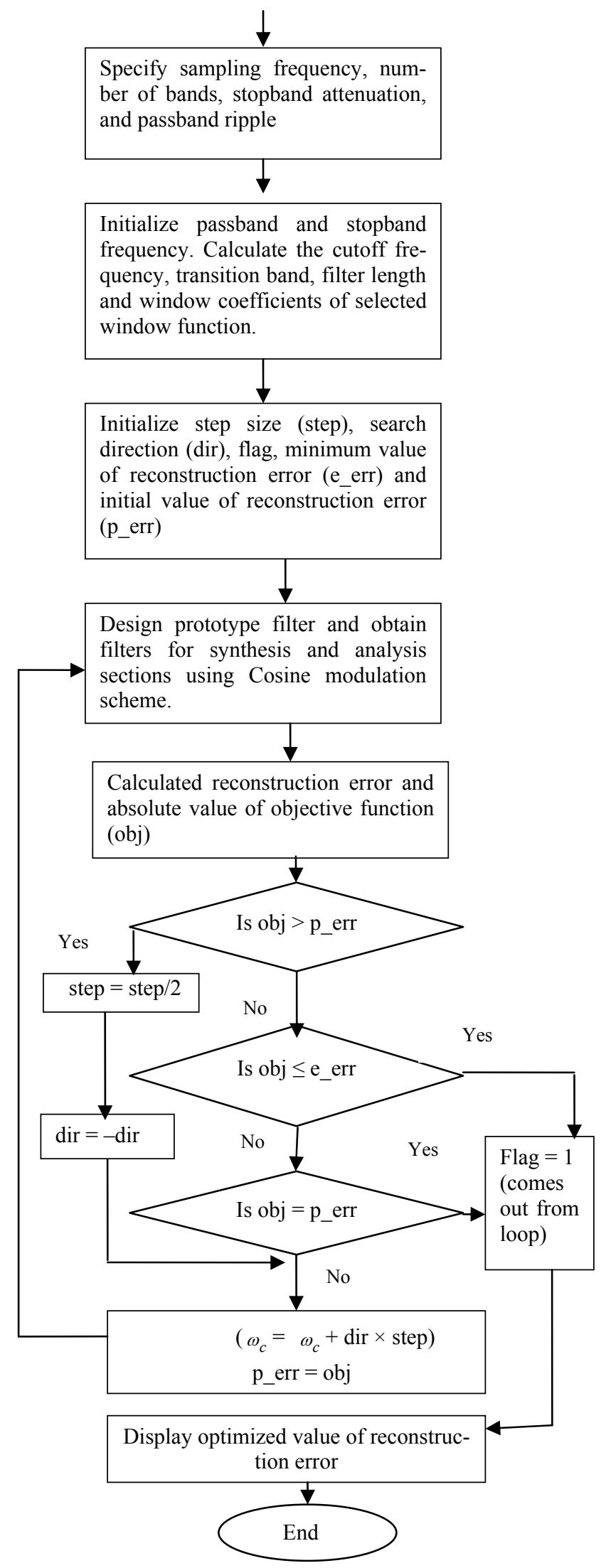

